\title{
The crystalline structures of the even alkanes hexane, octane, decane, dodecane and tetradecane monolayers adsorbed on graphite at submonolayer coverages and from the liquid $\dagger$
}

\author{
Thomas Arnold, ${ }^{a}$ Robert K. Thomas, ${ }^{a}$ Miguel A. Castro, ${ }^{b}$ Stuart M. Clarke, ${ }^{* c}$ \\ Loic Messe ${ }^{c}$ and Akira Inaba ${ }^{d}$ \\ ${ }^{a}$ Physical and Theoretical Chemistry Laboratory, University of Oxford, South Parks Road, \\ Oxford, UK \\ ${ }^{b}$ Instituto de Ciencia de Materiales de Sevilla, Avda. Americo Vespucio, Sevilla, Spain \\ ${ }^{c} B P$ Institute and Department of Chemistry, University of Cambridge, Madingley Rise, \\ Madingley Road, Cambridge, UK CB3 OHE \\ ${ }^{d}$ Department of Chemistry, Graduate School of Science, Osaka University, Toyonaka, \\ Osaka 560, Japan
}

Received 10th September 2001, Accepted 1st November 2001

First published as an Advance Article on the web 4th January 2002

\begin{abstract}
Neutron and X-ray diffraction have been used to structurally characterise the crystalline monolayer structures of hexane, octane, decane, dodecane and tetradecane adsorbed on graphite at sub-monolayer coverages and when coexisting with liquid alkane. The structures of all the molecules investigated at both coverages and low temperatures are isomorphous with rectangular unit cells of plane group pgg containing two molecules per cell. In both high- and low-coverage structures the molecules have their extended axes parallel to the surface. The plane of the carbon skeleton is found to be parallel to the graphite surface. The monolayers at sub-monolayer coverages are interpreted as uniaxially commensurate while those monolayers coexisting with the liquid are fully commensurate. Dodecane and tetradecane are exceptional: dodecane forms additional phases at high temperatures just prior to melting, at both low and high coverages. In these structures the molecules in the unit cell are parallel to each other with plane group $\mathrm{cmm}$. Tetradecane only forms a single phase at high coverages in which the molecules appear to be parallel and upright, similar to the dodecane high temperature, high coverage phase.
\end{abstract}

\section{Introduction}

The structure, composition and behaviour of alkanes, carboxylic acids and alcohols, adsorbed from the vapour, liquid or binary solutions to solid surfaces is the result of the delicate balance of adsorbate and surface interactions which often leads to a complex phase behaviour on the surface, quite different from that of the bulk adsorbates. For example, many systems are found to adsorb solid, ordered monolayers, even where the stable phase in the bulk system is a liquid or solution. ${ }^{1-6}$ It is important to identify and characterise such solid monolayers in detail, not only to understand the behaviour of pure adsorbates but also to understand the behaviour of mixtures where the relative structures of the pure materials can be a key factor. ${ }^{5-7}$

Physisorbed monolayers have been extensively studied by a variety of techniques including STM, ${ }^{3}$ calorimetry ${ }^{8,9}$ and isotherm measurements. ${ }^{10-12}$ More recently these techniques have been complemented by the application of incoherent elastic neutron scattering (IQNS) and X-ray and neutron diffraction (ND). ${ }^{1,8,9,13}$ Incoherent elastic neutron scattering is able to indicate the presence of solid monolayers coexisting with liquid adsorbate and to distinguish the behaviour of each component of multicomponent systems. The neutron diffraction technique is able to provide detailed crystallographic information about the solid monolayers.

$\dagger$ Electronic Supplementary Information available. See http:// www.rsc.org/suppdata/cp/b1/b108190j/
In this work we present detailed structural analysis of the solid monolayers formed by the even membered alkanes hexane (C6), octane (C8), decane (C10), dodecane (C12) and tetradecane $(\mathrm{C} 14)$ at both submonolayer and multilayer coverages using a combination of neutron diffraction and X-ray diffraction data.

\section{Experimental}

Only a brief outline of the diffraction techniques is presented here (see ref. 14 for a full description). The apparatus and procedures for such experiments have been described elsewhere. ${ }^{15}$ The instruments used for the neutron diffraction were $\mathrm{D} 20$ and D1B at the Institut Laue-Langevin, Grenoble ${ }^{15}$ with incident wavelengths of 0.242 and $0.252 \mathrm{~nm}$ respectively. The X-ray experiments were performed at the Department of Chemistry, Osaka University. The equipment there is of the symmetrical transmission type with $\mathrm{Cu}-\mathrm{K} \alpha$ radiation, as described previously. ${ }^{16,17}$ Scattering from crystalline twodimensional adsorbed layers gives rise to diffraction peaks ${ }^{18,19}$ with a characteristic saw-tooth line shape. The solution of such patterns can be used to determine the structure of the layer in a manner analogous to diffraction from three-dimensional crystals. Solution of such patterns can be assisted by consideration of close packing arguments and the possible plane groups available to molecules with particular point groups. ${ }^{17}$ The diffraction pattern from an adsorbed monolayer is obtained by subtraction of the scattering from the substrate alone from that of the substrate and adsorbed material toge- 
ther. At higher coverages where there is a solid monolayer coexisting with bulk fluid phase the very broad peak characteristic of a fluid phase can be distinguished and removed in a straightforward fashion. For X-ray measurements protonated materials were used. For the neutron experiments deuterated alkanes were used to minimise the incoherent scattering background that would have arisen from protonated samples.

In the determination of the structure of an adsorbed monolayer it is important to combine both X-ray and neutron data, particularly when the molecules contain significant number of hydrogen atoms such as for the alkanes described here. The scattering by X-rays is dominated by the heavier elements while the neutron patterns are sensitive to all the elements. In several cases the structural solution based only on X-rays has been erroneous as the symmetry of the hydrogen-containing parts of a structure have a lower symmetry than that of the heavy elements. ${ }^{20}$

The adsorbent used was recompressed exfoliated recompressed graphite Papyex (Le Carbone Lorraine). Several graphite samples were used, each characterised by adsorption of nitrogen and found to have specific surface areas of $14-31.6 \mathrm{~m}^{2}$ $\mathrm{g}^{-1}$. The deuterated alkanes were obtained from Cambridge Isotope Laboratories with quoted deuteration levels of
$>99 \%$. The graphite substrates were outgassed under vacuum in an oven at $350^{\circ} \mathrm{C}$ before known quantities of alkane were added as liquid by micro-syringe under an inert atmosphere of helium. When dosing the graphite it is convenient to know the approximate number of equivalent monolayers adsorbed. This can be estimated from the areas per molecule, taken from the work of Groszek, ${ }^{21}$ and the specific surface area of the graphite.

\section{Results}

\section{Low-coverage structures}

Fig. 1 presents the experimental (a) X-ray and (b) neutron scattering patterns from 0.8 monolayers of hexane, octane, decane, dodecane and tetradecane adsorbed on graphite. The neutron data were collected at $50 \mathrm{~K}$ and the X-ray data at $10 \mathrm{~K}$. The graphite background has been subtracted in preparing these figures. The features at $2 \theta=42^{\circ}$ and $2 \theta=26.6^{\circ}$ in the neutron and X-ray patterns respectively arise from imperfect subtraction of the graphite background. The neutron patterns have also been corrected for instability of

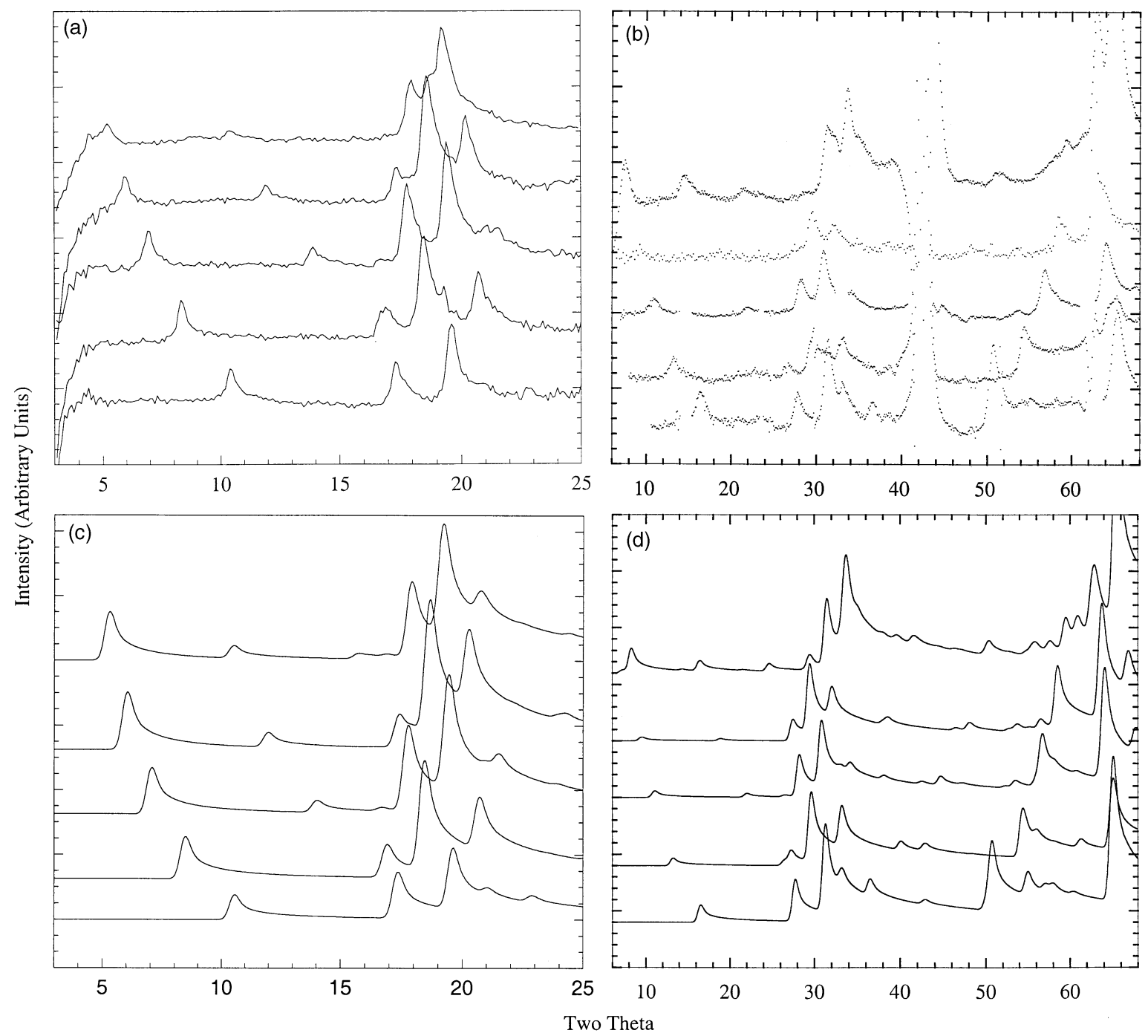

Fig. 1 (a) Experimental X-ray diffraction patterns from 0.75 monolayers of hexane (bottom), octane, decane, dodecane and tetradecane (top) adsorbed on graphite at low temperature. The scattering from the graphite substrate in the absence of any adsorbate has been subtracted in preparing this figure. (b) Experimental neutron diffraction patterns from 0.8 monolayers of hexane (bottom), octane, decane, dodecane and tetradecane (top) adsorbed on graphite at low temperature. The features at $2 \theta=42^{\circ}$ are regions of imperfect graphite subtraction. Calculated (c) neutron and (d) X-ray diffraction patterns from the solid monolayers of hexane (bottom), octane, decane, dodecane and tetradecane (top) on graphite. 
the multidetector on one of the diffractometers (D20). The peaks in these figures are then the diffraction from the adsorbed monolayers and have the characteristic asymmetric line shape expected from two-dimensional layers. ${ }^{18,19}$ In Fig. 1(b) the $x$-axis, 'two theta' refers to D20 data with a wavelength $0.242 \mathrm{~nm}$. D1B data has been included on this figure by calculating the effective two theta from the ratio of wavelengths of the two instruments.

The melting points of the layers, identified by the replacement of these sharp peaks by broad fluid-like reflections, are in excellent agreement with calorimetry data. ${ }^{12}$

\section{Coexisting liquids and solid monolayers}

Fig. 2 presents the experimental neutron scattering patterns from monolayers of hexane, octane, decane, dodecane and tetradecane adsorbed on graphite at higher coverages. The coverages and temperatures were 2 monolayers and $190 \mathrm{~K}$ (C6), 3 monolayers and $220 \mathrm{~K}(\mathrm{C} 8), 2$ monolayers and $250 \mathrm{~K}$ (C10), 3 monolayers and $270 \mathrm{~K}(\mathrm{C} 12)$ and 4 monolayers and $295 \mathrm{~K}$ (C14). For all the materials investigated the patterns from the monolayers were unchanged at coverages higher than 2 layers, indicating that these are the stable monolayer phases in the

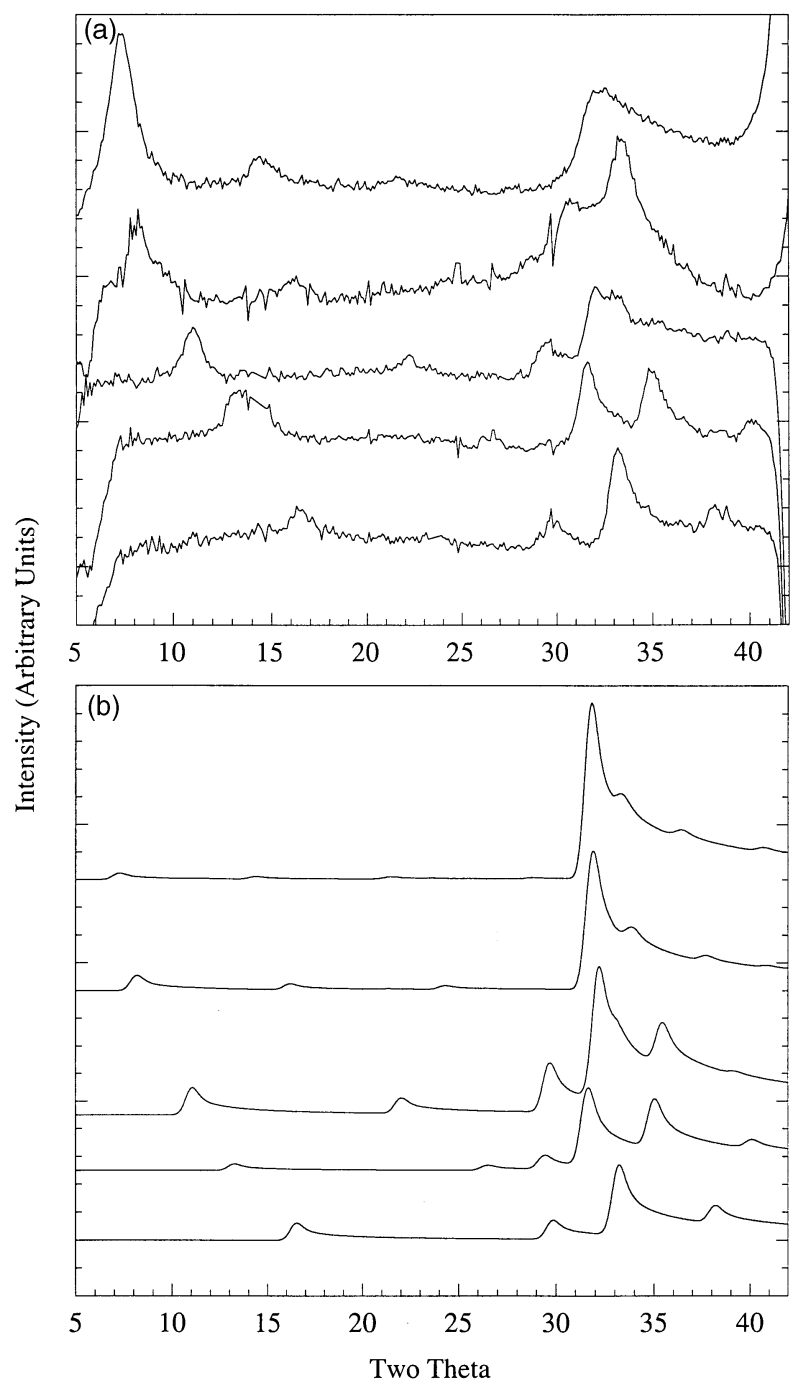

Fig. 2 (a) Experimental neutron diffraction patterns from the solid monolayers of hexane (bottom), octane, decane, dodecane and tetradecane (top) adsorbed on graphite at multilayer coverages coexisting with the liquid. The scattering from the bare graphite and that of the bulk fluid alkane have been subtracted in preparing this figure. (b) Calculated neutron diffraction patterns from the solid monolayers of hexane (bottom), octane, decane, dodecane and tetradecane (top) on graphite coexisting with the liquid. presence of bulk liquid, the temperatures of these patterns being above the bulk melting points (178 K (C6), $217 \mathrm{~K}$ (C8), $243 \mathrm{~K}(\mathrm{C} 10), 264 \mathrm{~K}(\mathrm{C} 12)$ and $279 \mathrm{~K}$ (C14)). In preparing these figures both the graphite background and the bulk fluid scattering have been subtracted as described previously. ${ }^{13}$ Features arising from imperfect subtraction and detector instability that are present in Fig. 1(b) are also present in this figure.

In marked contrast to the sub-monolayer coverages, the melting point of the solid monolayers which coexist with the liquid are higher than the bulk melting points, as described previously. ${ }^{13}$

\section{Dodecane}

Recent calorimetry and incoherent neutron scattering experiments have clearly indicated that dodecane shows an additional phase change with increasing temperature and with increasing coverage. ${ }^{12,22}$ Thus, there are four structures possible for adsorbed dodecane. The low-temperature patterns have been presented above. The neutron diffraction pattern from 0.75 monolayers of deuterated dodecane at a temperature of $220 \mathrm{~K}$ is shown in Fig. 3(a) and the pattern at 3 monolayers at $290 \mathrm{~K}$ is shown in Fig. 3(b). The principal difference been

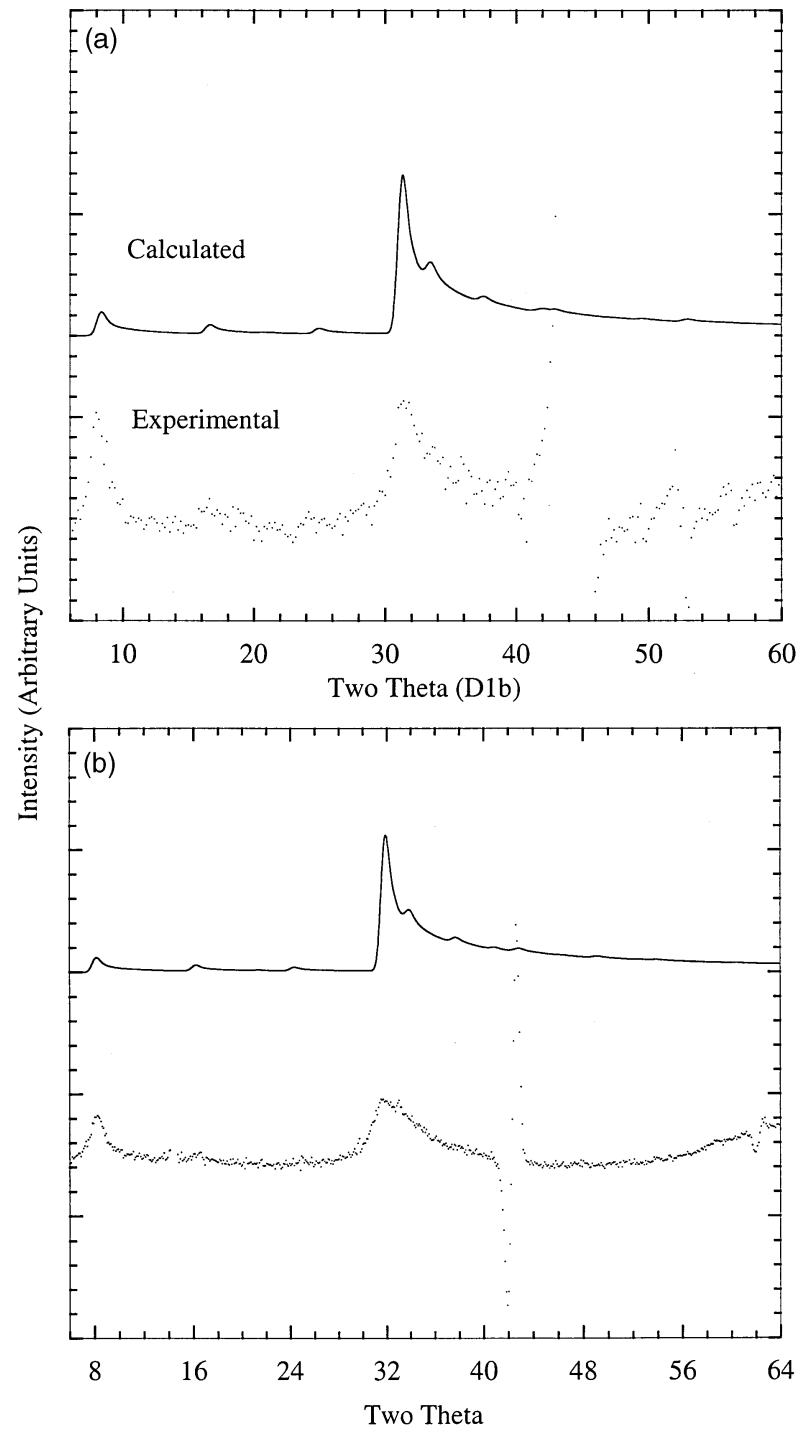

Fig. 3 (a) Experimental neutron diffraction patterns from the solid monolayer of dodecane adsorbed on graphite at high temperatures and submonolayer coverages. (b) Experimental neutron diffraction patterns from the solid monolayer of dodecane adsorbed on graphite at high temperatures at multilayer coverages. The calculated neutron diffraction patterns are also given in the figures. 


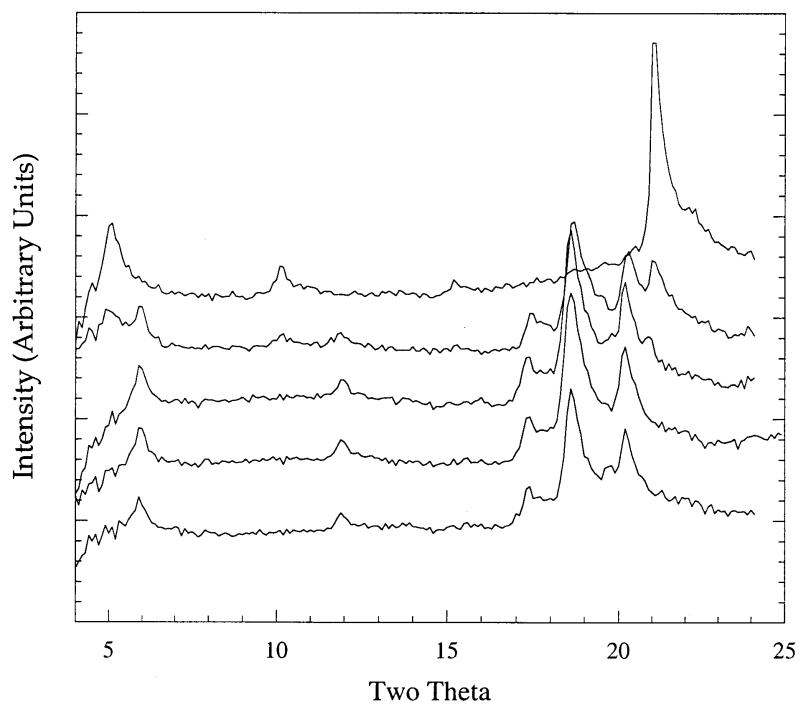

Fig. 4 X-ray diffraction patterns of dodecane at low temperature at increasing coverages of $0.6,0.75,0.84,0.90$ and 1.05 (top) monolayers.

the low- and high-temperature patterns is the loss of a number of peaks and a shift in the positions of others. On cycling the temperature it was found that the high-temperature to lowtemperature phase transition at high coverage exhibited hysteresis, indicating a first order transition. In keeping with traditional nomenclature the low- and high-temperature phases are referred to as phase II and phase I respectively.

Fig. 4 gives the X-ray diffraction patterns of dodecane at low temperature with increasing coverages of $0.6,0.75,0.84,0.90$ and 1.05 (top) monolayers. This figure clearly shows that there is coexistence of two phases over a significant coverage range between the sub-monolayer and high coverage monolayers, i.e. between 0.84 and 1.05 monolayers.

\section{Structural analysis}

Unlike conventional 3D crystallography, there are usually many less reflections in the experimentally determined diffraction patterns from adsorbed monolayers, and only a limited number of structural parameters can realistically be extracted. When fitting the experimental diffraction patterns we have therefore taken the molecular bond lengths and angles to be the same as in the bulk crystalline phases of the alkanes. $^{23}$ Additionally, the alkanes were assumed to be adsorbed in the all-trans configuration. The diffraction pattern for each trial structure was calculated as described previously. ${ }^{14,17,24}$ In this analysis we have adopted the usual convention of solving the patterns with the most highly constrained parameter set possible. In this approach we consider high symmetry rectangular unit cells first before moving to less symmetric and then oblique unit cells if solutions cannot be found. It is always easier to fit an experimental pattern by adding more variables.

In the following discussion we have grouped the structures according to their similarity. The low-temperature, low-coverage phases of hexane, octane, decane, dodecane and tetradecane, with the low-temperature, high-coverage phases of hexane, octane and decane, form a single isomorphic group. The other structures of dodecane and tetradecane are discussed separately.

\section{Herringbone structures}

It was found that a good fit to the experimentally determined peak positions of Fig. 1(a) and (b) for all the even alkanes and for Fig. 2(a) for hexane, octane and decane were (a)

(b)
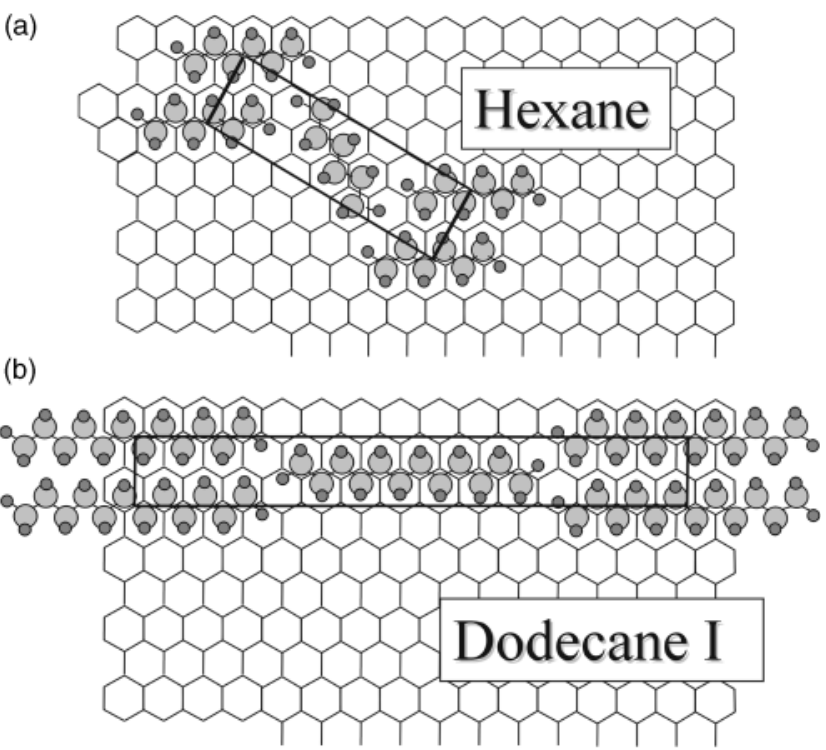

(c)

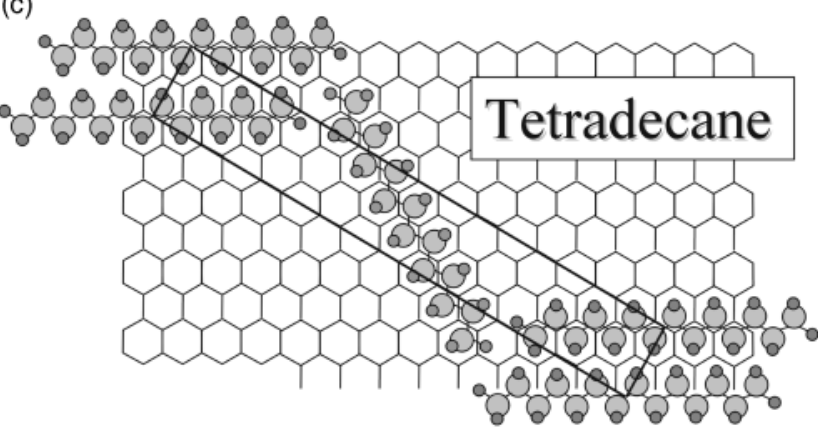

Fig. 5 Schematic representations of the monolayer crystal structures for (a) hexane, (b) dodecane I and (c) tetradecane adsorbed on graphite.

obtained with the isomorphic structures illustrated schematically in Fig. 5. The experimentally determined cell parameters are given in Table 1. Close packing considerations and the indexing of the experimental reflections can be used to determine the plane group of the adsorbed layers. ${ }^{17}$ The highest symmetry possible for the space group for the unit cell, centred, can be eliminated due to the presence of a strong $(2,1)$ reflection which is forbidden under centred symmetry (reflections $(h, k) h+k=$ odd are absent) for all the low-temperature phases. Furthermore, the systematic absences of reflections $(h, 0)$ with $h=$ odd and $(0, k)$ with $k=$ odd indicate a pgg space group. The symmetry related positions under pgg are given in Table 2. With this space group and the assumption of a fixed molecular conformation, the only adjustable parameters are the angles of orientation of the molecule in the cell. For any given molecule these two angles determine the intensities of the entire set of Bragg peaks. The calculated diffraction patterns are given in Fig. 1(c) and (d) and 2(b). The fractional coordinates of the repeating motifs in the unit cells are given as Electronic Supplementary Information in Tables S1-S12. $\dagger$ The fits to all the patterns based on this simple model are excellent and give structures isomorphic to that illustrated in Fig. 5(a), where the deuterium atoms of one molecule fit in the hollows of the adjacent chain. We discuss the accuracy of the determination of these structures below.

For hexane, octane, decane and dodecane the high-coverage, low-temperature phases are isomorphic with the low-coverage phases with the only difference being that the unit cell is compressed along the $b$-direction.

In the herringbone structures the angle the molecules make with respect to the $a$-axis of the unit cell (' $\mathrm{HB}$ ') is approxi- 
Table 1 Experimentally determined cell parameters for the adsorbed monolayers of the even alkanes and the closest commensurate structures. All cell parameters are in $\mathrm{nm}$. The length $\sqrt{ } 3 a_{\mathrm{g}}$ is $4.26 \mathrm{~A}$. The uncertainties in parameters $a$ and $b$ are estimated to be 0.02 and $0.01 \mathrm{~nm}$ respectively. $\mathrm{HB}$ is the angle between the molecular axis and the $a$-axis of the unit cell. Ax is the rotation angle of the molecule about its own axis

\begin{tabular}{|c|c|c|c|}
\hline \multirow[b]{2}{*}{ Alkane } & \multicolumn{2}{|l|}{ Low coverage } & \multirow[b]{2}{*}{ High coverage } \\
\hline & X-ray & Neutron & \\
\hline Hexane & $\begin{array}{l}0.54 \times 1.69 \\
0.54 \times 4 \sqrt{ } 3 a_{\mathrm{g}} \\
\mathrm{Ax}=0, \mathrm{HB}=25\end{array}$ & $\begin{array}{l}0.53 \times 1.69 \\
0.53 \times 4 \sqrt{ } 3 a_{\mathrm{g}} \\
\mathrm{Ax}=0, \mathrm{HB}=25\end{array}$ & $\begin{array}{l}0.49 \times 1.69 \\
2 a_{\mathrm{g}} \times 4 \sqrt{ } 3 a_{\mathrm{g}} \\
\mathrm{Ax}=0, \mathrm{HB}=25\end{array}$ \\
\hline Octane & $\begin{array}{l}0.544 \times 2.12 \\
0.544 \times 5 \sqrt{ } 3 a_{\mathrm{g}} \\
\mathrm{Ax}=0, \mathrm{HB}=28\end{array}$ & $\begin{array}{l}0.53 \times 2.12 \\
0.53 \times 5 \sqrt{ } 3 a_{\mathrm{g}} \\
\mathrm{Ax}=20, \mathrm{HB}=26.5\end{array}$ & $\begin{array}{l}0.49 \times 2.12 \\
2 a_{\mathrm{g}} \times 5 \sqrt{ } 3 a_{\mathrm{g}} \\
\mathrm{Ax}=30, \mathrm{HB}=25\end{array}$ \\
\hline Decane & $\begin{array}{l}0.547 \times 2.55 \\
5.47 \times 6 \sqrt{ } 3 a_{\mathrm{g}} \\
\mathrm{Ax}=0, \mathrm{HB}=29\end{array}$ & $\begin{array}{l}0.540 \times 2.55 \\
0.540 \times 6 \sqrt{ } 3 a_{\mathrm{g}} \\
\mathrm{Ax}=10, \mathrm{HB}=28\end{array}$ & $\begin{array}{l}0.51 \times 2.55 \\
0.51 \times 6 \sqrt{ } 3 a_{\mathrm{g}} \\
\mathrm{Ax}=0, \mathrm{HB}=29\end{array}$ \\
\hline Dodecane (Phase II) & $\begin{array}{l}0.546 \times 2.98 \\
0.546 \times 7 \sqrt{ } 3 a_{\mathrm{g}} \\
\mathrm{Ax}=0, \mathrm{HB}=30\end{array}$ & $\begin{array}{l}0.546 \times 2.98 \\
0.546 \times 7 \sqrt{ } 3 a_{\mathrm{g}} \\
\mathrm{Ax}=0, \mathrm{HB}=28\end{array}$ & $\begin{array}{l}0.426 \times 3.50 \\
\mathrm{Ax}=0, \mathrm{HB}=0\end{array}$ \\
\hline Tetradecane & $\begin{array}{l}0.555 \times 3.40 \\
0.555 \times 8 \sqrt{ } 3 a_{\mathrm{g}} \\
\mathrm{Ax}=0, \mathrm{HB}=33\end{array}$ & Composite pattern & $\begin{array}{l}0.445 \times 3.90 \\
\text { Axial disordered, } \\
\mathrm{HB}=0\end{array}$ \\
\hline Dodecane (Phase I) & $\mathrm{N} / \mathrm{A}$ & $\begin{array}{l}0.474 \times 3.52 \\
0.474 \times 14 a_{\mathrm{g}} \\
\text { Axial disordered, } \\
\mathrm{HB}=0\end{array}$ & $\begin{array}{l}0.450 \times 3.46 \\
0.450 \times 14 a_{\mathrm{g}} \\
\text { Axial disordered, } \\
\mathrm{HB}=0\end{array}$ \\
\hline
\end{tabular}

mately $30^{\circ}$ in all cases (see Table 1$)$. There are small variations between the different structures but these differences are close to the experimental error $\left( \pm 4^{\circ}\right)$.

It is interesting that the $a$-axis parameters of the unit cells of all the low temperature monolayers are close to integer multiples of $\sqrt{ } 3 a_{\mathrm{g}}(0.426 \mathrm{~nm})$, where $a_{\mathrm{g}}$ is the lattice parameter of the graphite $(0.246 \mathrm{~nm})$. However only at high coverage does the $b$-axis parameter appear to be related to the graphite lattice parameter $\left(2 a_{\mathrm{g}}\right)$. This result indicates that the monolayers are uniaxially commensurate at low coverages and fully commensurate at high coverages. Clearly there is a uniaxial compression in the $b$-direction on increasing the coverage. Table 1 also indicates small differences in the length of the unit cell in the $b$-direction between X-ray and neutron measurements at low coverages for samples notionally made up at the same coverage, although the $a$ parameter is the same. This indicates that the $b$-axis is strongly coverage dependent at coverages close to a monolayer. Such a coverage dependence suggests that the transition is a continuous one.

In the ideal commensurate structure $\mathrm{CH}_{2}$ groups sit in the graphite hexagons and the molecular axis makes an angle of exactly $30^{\circ}$ with the $a$-axis. The experimentally determined molecular orientation is therefore also consistent with a commensurate layer. The molecular orientation about the long axis of the molecules has been the subject of some debate. For all the alkanes investigated here the patterns from the low temperature phases indicate that the molecules lie on the surface with the plane of the carbon backbone parallel to the plane of the graphite. Although the calculated intensities of the observed peaks in the patterns are not very sensitive to this orientation, they define it to $\pm 20^{\circ}$. Fig. 6 presents examples of calculated scattering patterns for different molecular orientations demonstrating this sensitivity to the rotational position and clearly showing that the flat orientation of the molecules

Table 2 Symmetry operations of the pgg space group

\begin{tabular}{ll}
\hline$x, y$ & $-x,-y$ \\
$1 / 2+x, 1 / 2-y$ & $1 / 2-x, 1 / 2+y$ \\
\hline
\end{tabular}

represents a significantly better fit to the experimental data than an upright orientation.

Scanning tunnelling microscopy (STM) has also been used to investigate physisorbed monolayers of simple alkyl species on graphite. However, it is clear that such data should be treated with extreme caution both because interpretation of the experimental data is very complex and because it is now well established that the sweeping of the STM probe can perturb the delicate structures that are being imaged. Recent STM of much longer alkanes than studied here adsorbed on graphite have shown the appearance of crystalline monolayer structures with oblique unit cells. For such long molecules the positions of the peaks in the diffraction patterns are dominated by the large $a$-parameter, resulting in little difference in the scattering patterns between rectangular and oblique cells. However, for the much shorter molecules investigated, using the non-inva-

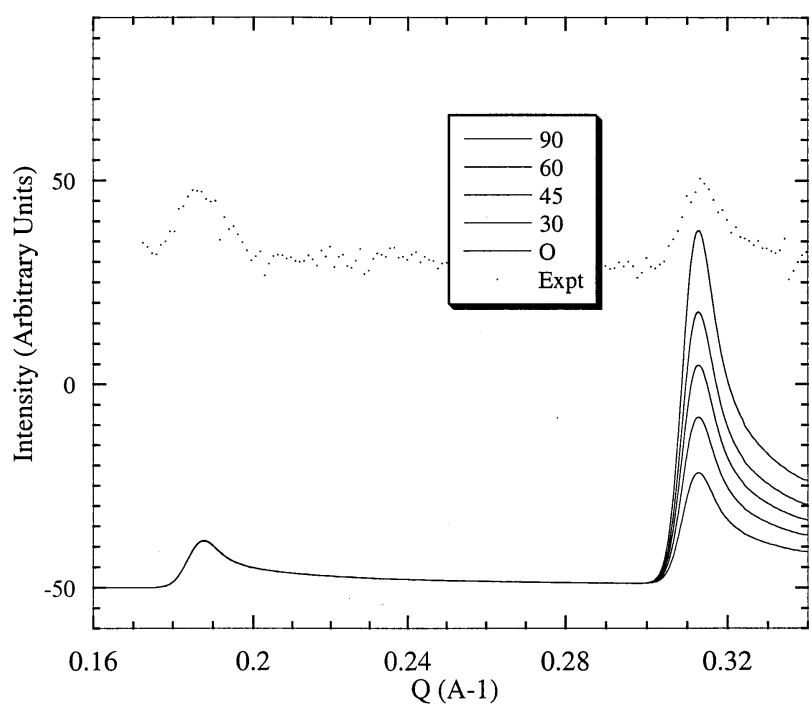

Fig. 6 Calculated neutron scattering patterns for different molecular orientations of adsorbed molecules in adsorbed monolayers. See text for discussion. 
sive technique of neutron scattering, the results clearly support the formation of rectangular cells and the distinction between rectangular and oblique unit cells is unambiguous.

\section{Phases of dodecane and tetradecane}

The low-coverage, low-temperature monolayer structure of dodecane is isomorphic with the other even alkanes discussed above. However, on increasing the temperature the experimental patterns change to give the new pattern of Fig. 3(a). The reflections of Fig. 3(a) can be indexed on a rectangular unit cell as given in Table 1 . The very limited number of reflections clearly indicates a highly symmetric unit cell with apparent plane group $\mathrm{cmm}$. The symmetry related atomic positions under $\mathrm{cmm}$ symmetry are given in Table 3 . For even alkanes this highly symmetric structure can only arise in certain molecular orientations, in particular if the molecules have the plane of the carbon backbone upright. Alternatively, if the molecules are rotating about their long axis then the time averaged structure will appear to be highly symmetrical. We are not able to distinguish these two alternatives from the intensities of the peaks.

Significantly the length of the unit cell $a$-axes is essentially double the length of the molecules. Hence we can conclude that the molecules do not lie in the herringbone arrangement found at low temperatures but are arranged parallel to each other in the structure shown schematically in Fig. 5(b).

Often, the appearance of a higher temperature solid phase is associated with an increase in symmetry following the introduction of some disorder. Significantly the intensities of the $(h, 0)$ peaks for this high-temperature structure are stronger relative to the $(h, k)$ than would be suggested by simple static models. This suggests significant disorder in the layer. Apart from the possibility of rotational disorder already mentioned above, there is the possibility of disorder associated with translational motion. Recent STM studies of longer alkanes adsorbed on graphite have also reported that the alkane molecules line up parallel to each other forming 'lamellae'. ${ }^{25} \mathrm{In}$ the STM work there were indications of a sliding of the lamellae. If this were the case than the periodicity characteristic of the lamellae would be expected to remain strong, i.e. the $(h, 0)$ reflections would remain strong. However, the general reflections of the type $(h, k)$ could be broadened or even disappear altogether. In the absence of other experimental information about the dynamics and spatial extent of this motion it is not possible to quantify it from the diffraction pattern.

The coverage-dependent X-ray patterns of Fig. 4 show the evolution of the high-coverage low-temperature structure of dodecane. Again, the a-parameter of the unit cell clearly indicates parallel molecules just as for the high-temperature, low-coverage phase. However, the $b$-parameter is slightly shorter at higher coverages, showing the compression evident for the herringbone structure of the other even alkanes. For this structure the symmetry is not as high as the low-coverage, high-temperature phase, indicating that the molecules are not disordered and intensity calculations suggest that the plane of the carbon backbone is within $20^{\circ}$ of being parallel to the surface, but we cannot determine the angle with any accuracy.

The high-coverage, high-temperature phase of dodecane appears to have the molecules all parallel, either with the plane of the backbone upright or rotationally disordered, similar to the low-coverage high-temperature structure. The disorder is

Table 3 Symmetry operations of the $\mathrm{cmm}$ space group

\begin{tabular}{ll}
\hline$x, y$ & $-x,-y$ \\
$1 / 2+x, 1 / 2+y$ & $1 / 2-x, 1 / 2-y$ \\
$x,-y$ & $-x, y$ \\
$1 / 2+x, 1 / 2-y$ & $1 / 2-x, 1 / 2+y$ \\
\hline
\end{tabular}

evident in a $b$-axis value larger than the commensurate value and in the loss of higher order reflections. However the hightemperature structures at low and high coverage differ slightly in that the high-coverage phase is slightly compressed. Detailed investigation of the high-coverage phase indicates that it expands continuously with increasing temperature, prior to melting, and contracts on increasing the coverage.

The low-coverage phase of tetradecane is isomorphic with all the other even alkanes at this coverage. However, the high coverage phase of tetradecane is found to adopt a similar structure to high-coverage, high-temperature dodecane with a parallel arrangement of the molecules. The neutron pattern of Fig. 1(b) is at a coverage where both phases coexist, similar to the coverage dependence seen with dodecane, Fig. 4. This highcoverage phase has a $b$-parameter larger than commensurate and no high order peaks. However, as described above for dodecane, we cannot reliably distinguish upright backbones from rotational disorder.

Some of the characteristics of the structures of high-coverage dodecane and tetradecane, are reminiscent of the rotator phases observed in bulk alkanes, although bulk rotator phases in even alkanes do not occur until C20. ${ }^{26-29}$ As mentioned above, specific calculations to distinguish the static $\mathrm{cmm}$ structure from a rotator phase were generally inconclusive.

\section{Discussion and conclusions}

The low-temperature monolayer structures of the even alkanes, octane to tetradecane, are found to be isomorphic, differing only in an expansion by an integral value of $0.426 \mathrm{~nm}$ for each additional $\mathrm{CH}_{2} \mathrm{CH}_{2}$ and a uniaxial compression on increasing the coverage above a monolayer. This structure is also isomorphic with those of adsorbed butane and hexane monolayers that have been previously reported based on neutron data only. 40

The molecules in nearly all the structures appear to have the planes of their carbon backbones parallel to the graphite. However, the experimentally determined patterns are not always particularly sensitive to the orientation of the alkanes about their long axis on the surface. This is particularly the case for dodecane where it appears that the carbon plane is parallel to the graphite surface at low temperature but may be upright or rotationally disordered in the high-temperature phase. The highcoverage phases of dodecane and tetradecane also differ from the majority of the structures in that the molecules are in a parallel arrangement. A recent STM study ${ }^{3}$ of the C27 alkane has identified lamellar structures with extended carbon chains oriented parallel to the graphite basal plane, in good agreement with our results. In the STM study the alkane chains are almost perpendicular to the lamellae, similar to the high-temperature phases of dodecane and tetradecane. In the homogolous series of linear alkanes it appears that odd alkanes and even alkanes of $\mathrm{C} 12$ or longer may adopt this parallel arrangement of molecules.

The crystallographic structure of all monolayers have pgg symmetry in agreement with the close-packing predictions of Kitaigorodskii. ${ }^{17}$ However, the detailed comparison with what is or is not the true close-packed structure is complicated by the effects of the commensurability with the underlying graphite. The complexity of these effects is well illustrated by the fact that the layers are commensurate along one direction and continuously variable up to commensurability in the other.

\section{Acknowledgements}

The authors thank UK EPSRC (TA), The Spanish DGICYT, The Leverhulme Trust (LM) and The Yamada Science Foundation (AI) for financial support and the Staff and scientists at the ILL and ISIS for beam time and technical assistance. 


\section{References}

1 M. A. Castro, S. M. Clarke, A. Inaba and R. K. Thomas, J. Phys. Chem. B, 1997, 101, 8878-8882.

2 M. Castro, S. M. Clarke, A. Inaba, T. Arnold and R. K. Thomas, J. Phys. Chem. Solids, 1999, 60, 1495-1497.

3 J. P. Rabe and S. Buchholz, Science, 1991, 253, 424

4 H. Taub, in The Time Domain in Surface and Structural Dynamics, ed. G. J. Long and F. Grandjean, Kluwer, Dordrecht, 1988, vol. 228.

5 M. Castro, S. M. Clarke, A. Inaba, T. Arnold and R. K. Thomas, Phys. Chem. Chem. Phys., 1999, 1, 5017-5023.

6 M. Castro, S. M. Clarke, A. Inaba, R. K. Thomas and T. Arnold, Phys. Chem. Chem. Phys., 2001, 3, 3774-3777.

7 M. A. Castro, S. M. Clarke, A. Inaba, R. K. Thomas and T. Arnold, J. Phys. Chem. B, 2001, 105, 8577-8582.

8 P. Epseau and J. White, J. Chem. Soc., Faraday Trans., 1997, 93, 3197-3200

9 P. Epseau, P. A. Reynolds, T. Dowling, D. Cookson and J. White, J. Chem. Soc., Faraday Trans., 1997, 93, 3201-3208.

10 G. H. Findenegg, J. Chem. Soc., Faraday Trans., 1972, 68, 1799.

11 G. H. Findenegg, J. Chem. Soc., Faraday Trans., 1973, 69, 1969.

12 S. M. Clarke, A. Inaba, T. Arnold and R. K. Thomas, J. Therm. Anal. Calorim., 1999, 57, 641-651.

13 M. A. Castro, S. M. Clarke, A. Inaba, T. Arnold and R. K. Thomas, J. Phys. Chem. B, 1998, 102, $10528-10534$.

14 R. A. Bucknall, S. M. Clarke, R. A. Shapton and R. K. Thomas, Mol. Phys., 1989, 67, 439
15 ILL (Institut Laue-Langevin, Grenoble, France, 1996).

16 S. M. Clarke, D.Phil., University of Oxford, 1986.

17 S. M. Clarke and R. K. Thomas, Mol. Phys., 1991, 72, 413-423.

18 J. K. Kjems, L. Passell, H. Taub, J. G. Dash and A. D. Novaco, Phys. Rev. B, 1976, 13, 1446.

19 B. E. Warren, Phys. Rev., 1941, 59, 693.

20 K. Morishige, Y. Tajima, S. Kittaka, S. M. Clarke and R. K. Thomas, Mol. Phys., 1991, 72, 395-411.

21 A. J. Groszek, Proc. R. Soc. London, Ser. A, 1970, 314, 473.

22 S. M. Clarke, A. Inaba, T. Arnold, R. K. Thomas and M. Castro, J. Phys. Soc. Jpn., 2001, 70 (Suppl. A), 300-302.

23 H. Mathisen, N. Norman and B. F. Pedersen, Acta. Chem. Scand., 1967, 21, 127-134.

24 K. Knorr, Phys. Rep., 1992, 214, 113.

25 R. Hentschke, L. Askadskaya and J. P. Rabe, J. Chem. Phys., 1992, 97, 6901-6909.

26 P. Espeau, Y. Hagety, M. A. Cuevas-Diarte and H. A. J. Oonk, in Proceedings of the XXI J.E.E.P., Rouen, 1995.

27 E. B. Sirota, H. E. King, H. H. Shao and D. M. Singer, J. Phys. Chem., 1995, 99, 798-804.

28 G. Ungar, J. Phys. Chem., 1983, 87, 689.

29 E. B. Sirota, H. E. King, H. H. Shao and D. M. Singer, J. Chem. Phys., 1993, 98, 5809.

30 K. W. Herwig, J. C. Newton and H. Taub, Phys. Rev. B, 1994, 50, $15287-15297$.

31 N. Norman and H. Mathisen, Acta. Chem. Scand., 1961, 15, 1755. 